EPiC Series in Language and Linguistics
Volume 3, 2018, Pages 72-78
LSP in Multi-disciplinary contexts of
Teaching and Research. Papers from the
16th International AELFE Conference

\title{
Consideraciones morfológicas en torno a la terminología del sector turístico aplicado a la lengua alemana con fines específicos
}

\author{
Beatriz Burgos Cuadrillero \\ Universidad de La Laguna, Campus Universitario de Guajara, San Cristóbal de La Laguna 38200, España
}

\begin{abstract}
Resumen
Bajo el techo de la lexicología es indiscutible la interrelación que se establece entre la morfología con la sintaxis y la semántica. En este sentido, el fenómeno de la formación de palabras ocupa siempre un importante lugar en la investigación lexicológica ya que genera modelos estables, es decir, nuevas formaciones de palabras tomando como punto de partida morfemas libres. Este hecho cobra especial importancia en una lengua como el alemán donde existe en torno a medio millón de palabras de las cuales unas pocas miles son palabras originales (Götze, $2005^{3}$ ) y el resto se han obtenido mediante el proceso de composición o a través de otros recursos morfológicos, de los cuales el más frecuente es la derivación. De tal manera que su estudio cobra plenamente sentido en el caso de las creaciones ad hoc.

La presente comunicación tiene por objeto realizar un análisis, desde el punto de vista morfológico, de la terminología en el sector turístico con la finalidad de descubrir el rol que desempeña la morfología léxica, estudio lingüístico que aplicaremos a la lengua alemana ya que los diferentes procesos de formación de palabras juegan el papel más importante en la ampliación del vocabulario de una lengua y, en lo que al alemán se refiere, este fenómeno es el ejemplo más claro de creatividad lingüística.
\end{abstract}

Keywords: Terminología, Léxico turístico, Morfología, Lengua alemana con fines específicos

\section{Introducción}

Un lenguaje especializado se caracteriza, principalmente, por su terminología lo que implica adquirir un léxico específico y necesario para el buen desarrollo profesional en un sector determinado, en nuestro caso, el turístico, sector ligado a disciplinas tan dispares como son la economía, la historia, el derecho, la geografía, el arte, la gastronomía, etc. Así pues, la heterogeneidad es una de las características fundamentales del corpus que conforma el léxico turístico ya que procede de diferentes y variadas disciplinas, hecho que lleva a Balboni a definir la lengua del turismo como un conjunto de "micro lenguas" que se interrelacionan entre sí y con diferente grado de notabilidad en los correspondientes ámbitos profesionales como, por ejemplo, la lengua de la hostelería en el sector de los hoteles y restaurantes (Calvi, 2005). 
En el caso concreto de la lengua alemana, una de sus particularidades es que gran parte del acervo léxico se ha obtenido mediante el proceso de composición o a través de otros recursos morfológicos, de los cuales el más frecuente es la derivación. En este sentido, el fenómeno de la formación de palabras ocupa siempre un importante lugar en la investigación lexicológica ya que genera modelos estables, es decir, nuevas formaciones de palabras tomando como punto de partida morfemas libres. Esta característica fundamental se traslada al sector turístico donde la creación de neologismos es una de sus particularidades principales gracias a la productividad de este fenómeno en alemán (Borrueco Rosa, 2005/06).

La formación de palabras engloba todo el conjunto de diferentes procedimientos para la formación de entidades léxicas nuevas partiendo de palabras ya existentes, entre los que destacan, fundamentalmente, dos: composición y derivación. Los resultados de estos procedimientos son productos compuestos y derivados (Wortbildungskonstruktionen, de ahora en adelante WBKs), nuevas palabras que se obtienen por diferentes motivos. Las palabras se componen de dos partes, significante y significado, y uno de los objetivos primordiales de la morfología es analizar el significante de la palabra descomponiéndola en sus constituyentes más inmediatos, es decir, en sucesivas etapas hasta llegar al 'morfema'.

La disciplina de la morfología tiene gran importancia al estudiar los diferentes procesos que integran la formación de palabras como un fenómeno independiente de la flexión de palabras y de la creación de palabras. Nuestro análisis se centra en el primer proceso teniendo en cuenta que se trata, siguiendo la tradición germanística, de la disciplina que estudia la formación de palabras y sus reglas, es decir, engloba aquellos procesos activos para generar nuevas palabras.

Nuestro objetivo es realizar un análisis, desde el punto de vista morfológico, de la terminología en el sector turístico con especial atención a la restauración, cuya finalidad es descubrir el rol que desempeña la morfología léxica, estudio lingüístico que aplicaremos a la lengua alemana ya que los diferentes procesos de formación de palabras juegan el papel más importante en la ampliación del vocabulario de una lengua y, en lo que al alemán se refiere, este fenómeno es el ejemplo más claro de creatividad lingüística.

\section{Morfología léxica o derivativa en la lengua alemana con fines específicos}

En el alemán contemporáneo se diferencian dos procesos principales de formación de palabras en base a la clase de morfemas sobre los que se sustentan: composición y derivación. A grandes rasgos, podemos definir el proceso compositivo como el resultado de la unión de, al menos, dos partes cuyos constituyentes son palabras o morfemas que se unen para formar una nueva y única palabra y puede haber diferentes tipos de composición como son la determinativa, la copulativa y la posesiva.

Frente al proceso compositivo se halla el derivativo conocido como derivación y que se define como una formación en la que uno de los constituyentes no puede aparecer de forma independiente, dando como resultado, en su uso más generalizado, la formación de palabras mediante afijos, es decir, mediante constituyentes que reciben diferentes denominaciones según su posición en la nueva palabra (prefijos, infijos, sufijos, circunfijos).

Es de notar que de ordinario ambos procesos son compatibles y que pueden aparecer en una misma formación como se refleja en la siguiente WBK: Nahrungsmittelunverträglichkeit. Dicho ejemplo muestra una combinación de elementos no homogéneos, es decir, de formaciones híbridas donde por una parte, aparecen palabras compuestas como Nahrung-s-mittel, y, por otra parte, se integran otros procesos de formación de palabras como la derivación mediante sufijos y prefijos que se refleja en la unidad léxica Un-ver-träg-lich-keit.

A este respecto, hay lingüistas como Kauffer que, más allá del tipo de procedimiento que 
unánimemente se considera como composición, esto es, la formación de un nuevo ítem léxico a partir de la combinación de dos o más piezas que funcionan como formas libres, establece una diferencia funcional entre composición y derivación haciendo alusión a una metáfora culinaria donde identifica las composiciones con los platos centrales, básicos en la gastronomía alemana y las derivaciones con los que giran en torno a ellas: "Komposita bezeichnen die Speisen, sind so etwas wie der "zentrale Bezeichnungskern", bleiben bei der Gastronomie; Derivate kreisen um diesen Bezeichnungskern, erlauben sich einen "abstrakten Kommentar" über die Speise, gehen zur "Meta-Gastronomie" über." (Kauffer cit. en Fleischer, Helbig y Lerchner, 2001).

A continuación, realizaremos un análisis descriptivo y taxonómico de la terminología en el sector turístico de la restauración prestando atención a los dos procedimientos más importantes en torno a los cuales gira la morfología derivativa alemana.

\subsection{La terminología en el sector turístico de la restauración: el proceso compositivo}

La finalidad de la composición es formar una nueva palabra, en nuestro caso por norma general, de categoría sustantiva por lo que necesariamente el segundo componente es un sustantivo y el primero puede ser una categoría gramatical de diferente índole, ya sea un sustantivo, un verbo, un adjetivo, un adverbio, un pronombre, un numeral o una preposición.

En el proceso de composición, la WBK está formada como mínimo por dos elementos léxicos y se divide, principalmente, en dos clases en base a la relación que establecen entre sí los miembros de la misma: 1) determinativa cuando la relación es de subordinación, es decir, un constituyente inmediato depende de otro matricial; 2) copulativa cuando la relación es paratáctica, esto es, de coordinación entre ambos constituyentes. Aunque hay que tener en cuenta que en cualquiera de los casos tanto el género como la clase de la palabra viene determinada por el segundo miembro.

La composición afecta especialmente al sustantivo y al adjetivo y, en menor medida, al adverbio. De entre estas clases de palabras que acabamos de presentar, cobran especial relevancia en nuestro corpus los sustantivos obtenidos mediante el proceso de composición y, especialmente, mediante la composición determinativa.

A este respecto, en los compuestos determinativos el cambio del orden de los elementos siempre está unido a un cambio de significado, por ejemplo, Fassbier $\neq$ Bierfass. Sin embargo, a diferencia de este ejemplo donde el orden de los elementos sí altera el significado del producto, los compuestos de tipo copulativo son formaciones cuyos constituyentes tienen una misma naturaleza, es decir, pertenecen a la misma clase de palabras y mantienen una relación paratáctica. Esta característica se refleja en que el primer miembro no determina al segundo, de tal manera que los elementos se pueden cambiar sin que varíe el significado de la formación resultante como podemos observar en los siguientes ejemplos: süßsauer $=$ sü $\beta$ und sauer; lauwarm = lau und warm.

En el corpus analizado ${ }^{1}$, la mayoría de los términos se clasifican como compuestos determinativos formados por dos constituyentes donde, tanto la palabra fundamental como la determinante, son de categoría sustantiva como observamos en los siguientes ejemplos:

Tabla 1. La terminología en el sector turístico de la restauración: la composición determinativa.

La composición determinativa: sustantivo + sustantivo

\footnotetext{
${ }^{1}$ Los ejemplos han sido tomados de Kerndter, 2007.
} 
En el campo de los compuestos determinativos, el lenguaje culinario cuenta con otros términos cuya palabra fundamental es un sustantivo unida a una palabra determinante de categoría adjetival: Dickmilch, Feingebäck, Frischkäse, Glattbutt, Hartkäse, Hartweizen, Jungente, Jungschwein, Magermilch, Mürbeteig, Rohkost, Rohschinken, Rohwurst, Sauerkirsche, Sauerkraut, Sauermilch, Süßkartoffel, Süßspeise, Süßstoff, Trockenerbsen, Trockenfrüchte, Vollmilch, Wildente, Wildkräuter, Wildragout, Wildschwein. Es de destacar también una serie de compuestos ligados a la gama cromática como son Blaubeere, Blauleng, Grünkohl, Rotbarbe, Rotbarsch, Rotbrassen, Rotkohl, Weißkohl.

La palabra determinante de esta serie de compuestos también la puede constituir un verbo como, por ejemplo, backen (Backpflaume, Backschinken, Backwerk), braten (Bratapfel, Brathähnchen, Bratkartoffeln, Bratwurst), dörren (Dörrfleisch, Dörrobst, Dörrpflaume), hacken (Hackbraten, Hackfleisch) o räuchern (Räucherfisch, Räucherschinken, Räucherspeck, Räuchertofu), entre otros. Asimismo, hay una serie de compuestos determinativos con un nombre propio como palabra determinante: Chinakohl, Jakobsmuschel, Johannisbeere, Petersfisch, Venusmuschel, Viktoria(see)barsch.

Sin embargo, en el léxico gastronómico también se encuentran compuestos determinativos con un adjetivo como palabra fundamental como Eigelb o Eiweiß o compuestos determinativos con tres constituyentes como son Blätterteiggebäck, Blätterteigpastete, Feinkostsalat, Fruchtsafteis, Gänseleberpastete, Kalbsnierenbraten, Pfefferminztee, Regenbogenforelle, Rinderschmorbraten, Sojabohnensprossen, Sonnenblumenkerne, Sonnenblumenöl, Vollkornbrot, Vollkornmehl. 


\subsection{La terminología en el sector turístico de la restauración: el proceso derivativo}

La derivación es junto con la composición el camino más importante para la formación de palabras en lengua alemana. Se trata de un proceso formado por la unión de un morfema fundamental o libre que constituye el núcleo -y, al mismo tiempo, actúa como palabra base- junto con un morfema ligado de formación que muestra una diferente naturaleza según su posición (prefijo, infijo, sufijo, circunfijo, transfijo); este proceso da como resultado el derivado.

La derivación está formada por una base que se transforma en una palabra derivada gracias a dos procesos fundamentalmente: derivación explícita (derivación con afijos) y derivación implícita. En los procesos derivativos se dan tanto mecanismos de derivación explícita como implícita integrados por un radical como morfema fundamental más los morfemas de relación, aunque debemos resaltar que las derivaciones analizadas son explícitas y destacan, en cuanto a frecuencia, los mecanismos sufijales.

En el análisis de la terminología culinaria, los derivados prefijales constituyen un pequeño grupo formado por una serie de verbos prefijados mediante ab- (abhängen, abkühlen), an- (anbrennen, anmachen), auf- (aufwärmen), be- (begleiten), ein- (einfrieren), er- (erhitzen), über(überbacken/überkrusten), um- (umdrehen, umrühren), ver- (verdauen, verdünnen, verdunsten, veredeln, verfeinern, verpacken, verschütten) o zer-(zerkleinern).

Sin embargo, como hemos mencionado anteriormente, la mayoría de los derivados se genera mediante el proceso explícito a través de la sufijación. Por este motivo, según el tipo de sufijo que se añada a la raíz del derivado surgen diferentes clases de palabras desde el punto de vista gramatical. En nuestro corpus son frecuentes los sufijos sustantivales en -ung y -keit: Erfrischung, Füllung, Süßigkeit, así como el sufijo -chen para generar diminutivos: Bällchen, Brötchen, Hähnchen, Hörnchen, Plätzchen, Radieschen, Schiffchen, Törtchen.

En cuanto a los derivados adjetivales, el sufijo más productivo es -ig que da lugar a adjetivos como, por ejemplo, blutig, cremig, durchsichtig, faserig, flüssig, fruchtig, knusprig, körnig, mehlig, obergärig, ölig, ranzig, saftig, sahnig, sämig, samtig, sprudelig, untergärig. Aunque también son frecuentes los adjetivos derivados en -lich como son appetitlich, bekömmlich, köstlich, natürlich, pflanzlich, säuerlich, schädlich; en -isch: biologisch, einheimisch, frisch, makrobiotisch, ökologisch, vegetarisch o en -bar: haltbar. En este grupo también destacan aquellos productos derivados mediante sufijoides como son los términos acabados en -reich: abwechslungsreich; en -arm: fettarm; en -frei: glutenfrei, koffeinfrei, laktosefrei o en -los: salzlos.

Asimismo, hay que destacar los derivados participiales que contribuyen ampliamente a engrosar el acervo léxico adjetival, tanto el participio de presente como el participio de pasado, siendo más frecuente en el sector gastronómico el participio de pasado. Véase: ausgeglichen, ausgezeichnet, gekörnt, gemahlen, gemischt, geräuchert, geröstet, ungespritzt o vorgekocht.

Por último, mediante el proceso derivativo también se genera una serie de derivados producto del proceso prefijal y sufijal en un solo término como, por ejemplo, verdaulich y verderblich siendo el resultado, en este caso, de adherir a la raíz el prefijo ver-y el sufijo adjetival -lich. En el caso del adjetivo unverdaulich, se observa la doble prefijación mediante un- que le aporta el contenido semántico de negación.

\subsection{La terminología en el sector turístico de la restauración: otros procesos formativos}

Junto con los dos grandes ejes en torno a los cuales gira la formación de palabras alemana, es decir, la composición y la derivación, existen otros procesos de formación de palabras como son la contaminación, la abreviación o los préstamos. En nuestro caso, el proceso más productivo son los préstamos aunque también se pueden encontrar en el lenguaje gastronómico siglas como $\mathrm{H}$ - 
Milch.

En cuanto a los extranjerismos, gracias a los que tiene lugar una destacada ampliación del vocabulario, el léxico de la restauración se caracteriza, principalmente, por los galicismos. En este grupo algunos términos han mantenido su forma original como son Aubergine, Barbe, Bonbon, Bouillon, Champignon, Chicorée, Creme, Dorade, Estragon, Farce, Filet, Fond, Fondant, Frisée, Gelee, Limette, Mandarine, Marinade, Medaillon, Mirabelle, Omelette, Orangeat, Pommes frites, Porree, Poularde, Praline, Raffinade, Ragout, Reineclaude, Roulade, Tomate, Vanille. ${ }^{2}$

Sin embargo, otros términos han sufrido diferentes procesos de germanización tanto a nivel ortográfico adoptando, especialmente, el cambio $\mathrm{k}<\mathrm{c}$ (Aspik, Biskuit, Frikassee, Kalmar, Karamell, Krokette, Rokambole), como a nivel gramatical germanizando verbos mediante la terminación -ieren (blanchieren, dekantieren, farcieren, flambieren, gelieren, gratinieren, kandieren, marinieren, tranchieren).

Por otro lado, la lengua alemana en el sector gastronómico cuenta también con una serie de anglicismos que han manteniendo el término de la lengua original (Beefsteak, Cocktail, Grapefruit, Grill, Pollack, Pudding, Sago, soft, vegan) o, en otros casos, se han germanizado (Keks $<$ Cakes). Además, el alemán cuenta con préstamos, normalmente germanizados, procedentes de la lengua italiana (Brokkoli, legieren, Limone, Marzipan, Sardelle, Sellerie, Zitrone, Zucchini), del portugués (Ananas, Banane), del español (Avocado, Oregano, Pepino) o del holandés (Anchovis, Bambus, Brasse, Waffel). Otros términos como Gulasch tienen su origen en el húngaro, Joghurt en el turco o Soja y Tofu en el japonés.

Entre otros procesos formativos también podemos destacar la formación de grupos nominales por adhesión de adjetivo como, por ejemplo, rote Bete, grüne Bohnen, dicke Bohnen, weiße Buttersoße, weiches Ei, rote/schwarze Johanissbeere, koffeinfreier Kaffee, gefüllte Kalbsbrust, rote Rübe, rundes Scheibchen, gefüllter Windbeutel; así como una serie de grupos nominales por adhesión de complementos: aus biologischem Anbau, nach Größe, auf der Grundlage von, eine Hand voll, von der Jahreszeit abhängig, mit/ohne Kohlensäure, eine Pfanne voll, im Ring, im Teigmantel.

\section{Conclusiones}

Los diferentes procesos de formación de palabras juegan el papel más importante en la ampliación del vocabulario de una lengua y, en lo que al alemán se refiere, este fenómeno es el ejemplo más claro de creatividad lingüística tanto en la lengua alemana general como en el sector de la restauración.

En el ámbito turístico, con especial atención al léxico gastronómico, la lengua alemana se caracteriza por un elevado número de neologismos como consecuencia de la productividad de los diferentes procesos de formación de palabras, especialmente, del proceso compositivo. Sin embargo, también son frecuentes términos derivados, sobre todo, por sufijación, y préstamos, fundamentalmente, galicismos.

\section{Referencias}

Borrueco Rosa, M.A. (2005/06). El discurso turístico: estudio lingüístico aplicado a la enseñanza de la traducción. Hieronymus Complutensis 12, 69-78. Recuperado de http://cvc.cervantes.es/lengua/hieronymus/pdf/12/12_069.pdf

\footnotetext{
${ }^{2}$ Estos sustantivos han adoptado las normas ortográficas de la lengua alemana, en concreto, nos referimos a la letra mayúscula al inicio de palabra. Los términos Creme, Gelee, Medaillon, Porree, Praline y Ragout han eliminado la tilde original; sin embargo, otros como Chicorée o Frisée la han mantenido.
} 
Calvi, M.V. (2005). El español del turismo: problemas didácticos. IDEAS (F-H Heilbronn), 1, 1-7. Recuperado de http://www.academia.edu/460041/El_Espa\%C3\%B1ol_Del_Turismo_Problemas_Did\%C3\%A1cticos

Duden Online Wörterbuch. Recuperado de http://www.duden.de/

Fleischer, W., Helbig, G. y Lerchner, G. (Eds.) (2001). Kleine Enzyklopädie: Deutsche Sprache. Frankfurt am Main, Alemania: Editorial Peter Lang.

Götze, L. $\left(2005^{3}\right)$. Das Wort. En L. Götze y W.W.B. Hess-Lüttich, Grammatik der deutschen Sprache (pp. 19-374). München, Alemania: Wissen Media Verlag.

Kerndter, F. (Ed.). (2007). Praxiswörterbuch. Internationale Küche. München: Langenscheidt Fachverlag GmbH. 